\title{
Surgery in amoebic colitis
}

\author{
D. STEIN AND SIMMY BANK
}

From the Departments of Surgery and Medicine and Gastro-intestinal Clinic, Groote Schuur Hospital and University of Cape Town, Observatory, Cape Town, South Africa

SUMMARY The feasibility of surgery in amoebic colitis is presented by illustrative case histories in two patients. Subtotal colectomy and ileostomy were carried out for fulminating colitis in the one and exteriorization, excision, and subsequent re-anastomosis of a localized perforated area of bowel in the other.

It is suggested that the place of surgery in amoebic colitis should be reappraised, particularly with the advent of modern medical and surgical methods now available. The indications for contemplating surgery in abdominal amoebiasis are outlined and a workable approach is presented.

The majority of patients with severe amoebic colitis respond rapidly and effectively to conservative medical measures. Surgical intervention is usually reserved for free colonic perforation or the drainage of intraperitoneal abscesses (Cope, 1920; Evans, 1925; Ochsner and Debakey, 1942; Wilmot, 1950; Barker, 1958; Wilmot, 1962; Peláez, Villazón, and Zaraboso, 1966). Apart from these two indications, surgery has usually been regarded as meddlesome and, in fact, contraindicated in amoebic colitis. The teaching that the colon resembles 'wet blotting paper' and is therefore hazardous to handle has resulted in a defeatist attitude towards operative procedures. Although isolated reports of colectomy and drainage of abscesses have been published, most textbooks disregard surgery completely (Theron, 1947; Barker, 1958).

The purpose of this article is to demonstrate that surgery is feasible in amoebic colitis, to discuss the surgical indications in colonic amoebiasis, and to provide reasons for the desirability of surgical intervention in some cases of fulminating amoebic colitis.

Received for publication 25 May 1970.

\section{Case Reports}

CASE 1

The patient, an African male of 36 years, was apparently quite well until one month before his admission to hospital on 4 July 1968 when he developed pain in the right upper quadrant radiating to the right loin and back. Four days before admission, he developed diarrhoea with blood and mucus in the stools associated with generalized cramp-like abdominal pains. He was given a 'potent enema' by two of his friends to help clear his bowel, after which he became increasingly ill; the enema was later established to consist of soap and water only. There was no past history of bowel upset and he had not been in contact with anyone with diarrhoea.

Examination on admission revealed him to be an ill patient with mild dehydration. The pulse was 120 , temperature $99^{\circ} \mathrm{F}$, and blood pressure $120 / 90$. There was diminished air entry and dullness at the base of the right lung and diffuse ronchi scattered throughout both lung fields. The abdomen was slightly distended but no masses were palpable. 'Punch' tenderness was present 
over the liver and bowel sounds were present. Rectal examination was difficult due to marked tenderness. The other systems were normal. The haemoglobin was $9.5 \mathrm{~g} \%$, ESR $11 \mathrm{~mm} /$ hour, and white cell count $3,500 \mathrm{~mm}^{3}$. Examination of the stools showed blood and mucus but no amoebae were visible on microscopy. Despite the negative stool examination, the diagnosis of amoebic colitis was favoured but typhoid, bacillary dysentery, or subphrenic abscess were also considered. He was treated with penicillin and streptomycin and intravenous fluids while being further investigated. On 6 July 1968 he suddenly developed generalized abdominal pain and his condition deteriorated rapidly. Examination at that time revealed gross abdominal distension and possible free fluid in the abdomen. Generalized tenderness was present with guarding but there was no true rigidity. A straight radiograph showed a dilated transverse colon and fluid levels in the small bowel (Fig. 1). Proctoscopy at that stage revealed a complete slough of the rectal mucosa. There was no normal mucosa to be seen and the slough extended to the mucocutaneous junction. Examination of the slough again failed to reveal any amoebae on microscopy. The proctoscopic findings appeared to substantiate amoebic colitis which had been aggravated by an enema given just before his admission. It was suspected that the slough involved the whole colon and that he had impending peritonitis.

Laparotomy was performed the same evening through a left paramedian incision. A large amount of turbid free peritoneal fluid was found. The whole colon was wrapped up in the omentum.

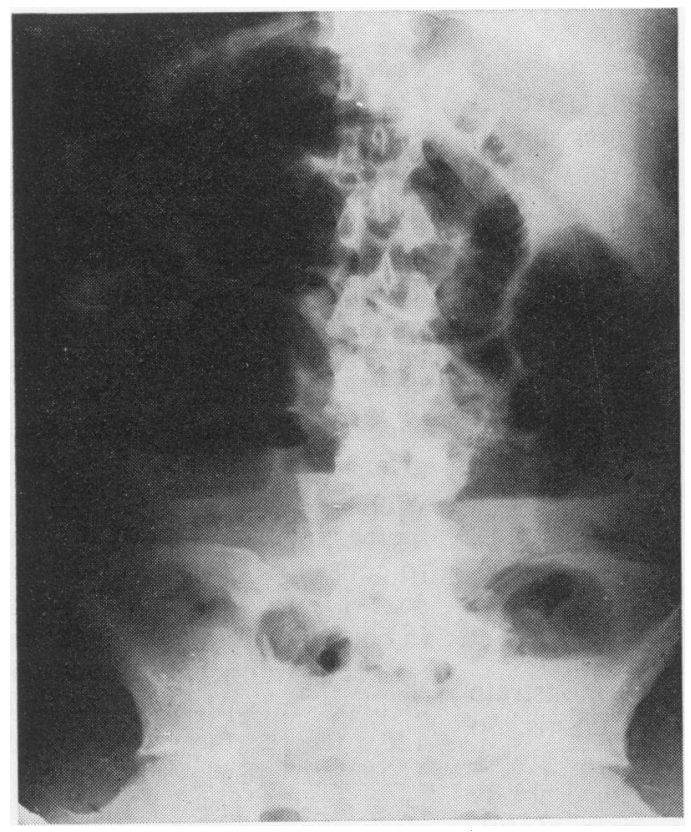

Fig. 1 Erect radiograph of the abdomen in case 1 showing a dilated large and small bowel in fulminating amoebic colitis.
The descending colon was stuck to the posterior abdominal wall. Several necrotic patches were? present in the rectum and sigmoid colon but there was no free perforation. The colon was $\overrightarrow{\vec{F}}$ opened at the hepatic flexure and the mucosa in the area had sloughed and this lesion had extended? more proximally, but to a lesser extent. The $\frac{\overline{\bar{c}}}{\bar{c}}$ upper rectum, sigmoid, descending and transverse ${ }_{\widetilde{\sigma}}$ colon were dissected free and excised. The $\varrho$ rectal stump was loosely closed with catgutes sutures and replaced in the peritoneal cavity. $\overrightarrow{0}$ Because of bleeding from the inflamed, raw retroperitoneal area the colon was resected as $\bar{\sigma}_{\sigma}$ far as the hepatic flexure only. The ascendinge

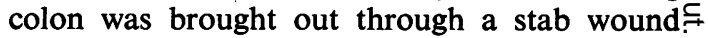
in the right upper quadrant as a colostomy. The $\overrightarrow{ }$ mucosa was necrotic at this area but the serosa was still intact. Several drains were inserted into 0 the peritoneal cavity and the wound closed. On $₫$ opening the resected transverse and the des-음 cending colon the whole mucosa was seen to besloughed, no viable islands of mucosal tissue $Z$ being present. The histology report showed ' $\mathrm{a}$ ⿸广 specimen consisting of large bowel measuring 3 approximately $44 \mathrm{~cm}$ long. The peritoneal surface $\Phi$ is thickened and partly covered by fibrinous exudate. The proximal portion of the bowel is dilated, measuring $11 \mathrm{~cm}$ in diameter, whilst the. distal portion is $7 \mathrm{~cm}$ in diameter. The mucosa is diffusely covered by a necrotic membranous type of lesion but very occasional areas of normalo tissue intervene suggesting that this is severe $\stackrel{0}{\circ}$ amoebic dysentery. The histology confirms å severe florid amoebic dysentery with vast numbers $\overrightarrow{\vec{O}}$ of amoebae in the tissue.'

Emetine hydrochloride, $1 \mathrm{~g}(65 \mathrm{mg})$ daily, chloroquine diphosphate, $250 \mathrm{mg}$ intramuscu-0. larly, and intravenous terramycin was started once receiving the histological report. Despite this therapy, the patient had a stormy postoperative 3 course and on 11 July 1968 it was noted that the mucosa at the colostomy site (where one small $\frac{1}{3}$ island of viable mucosa had previously been present) had now completely sloughed and it was 5 decided to remove the remaining colon. The $\frac{}{0}$ previous laparotomy wound was opened and this revealed necrotic patches of caecum and ascending $N$ colon as well as four gangrenous patches of the terminal ileum in its distal 18 inches. The colon and involved ileum were excised and an ileostomy was ${ }_{\sigma}^{\omega}$ fashioned. After the operation the patient required intensive supportive measures, including correction of the acidosis, isoprenaline infusions, $\varrho$ central venous pressure monitoring, and electro- lyte replacement therapy. He discharged large quantities of pus from the rectal stump and $\underset{\mathbb{D}}{\mathbb{D}}$ drainage wounds.

The histology report of the second operation $\stackrel{\mathbb{2}}{\circ}$ showed 'a specimen consisting of proximal part of the ascending colon, caecum, appendix, and $\delta$ distal ileum. The colon measured $20 \mathrm{~cm}$ and the ileum measured $30 \mathrm{~cm}$. The surface has a haemorrhagic appearance and in areas appears to be 
covered by fibrinous exudate. The appendix appears necrotic. The wall in areas appears thickened while in others it is haemorrhagic and viable. In the lumen there is a slough which involves the ileum proximally but at the distal line of excision it is absent. The whole of the caecum contains the slough. Cut section of the appendix shows necrotic material within its cavity. A severe amoebic infection of the colon, appendix, and distal ileum is present. The destruction of the bowel wall in areas has reached the serosa while in other areas has advanced as far as the muscularis. In no section examined histologically is the mucosa seen. The amoebae are extremely plentiful in all the sections. In addition, there is a local peritonitis.'

The ileostomy functioned satisfactorily from the outset despite the formation of a small bowel and rectal stump fistula. These fistulae closed spontaneously after several days of antibiotic therapy. The patient improved slowly and on 15 August 1968 a large liver abscess was drained under a local anaesthetic through the eleventh intercostal space in the posterior axillary line. An underwater drain was inserted. Microscopy of the pus obtained revealed the presence of active amoebae despite a full course of emetine. Radio-opaque dye was inserted via the tube into the abscess cavity confirming the presence of the abscess within the liver substance. A repeat course of emetine hydrochloride, 1 grain daily, was commenced but the patient continued to discharge a large amount of pus from the liver drain. On 25 August 1968, metronidazole, $800 \mathrm{mg}$ four times daily, was commenced and from then on the patient made rapid strides towards

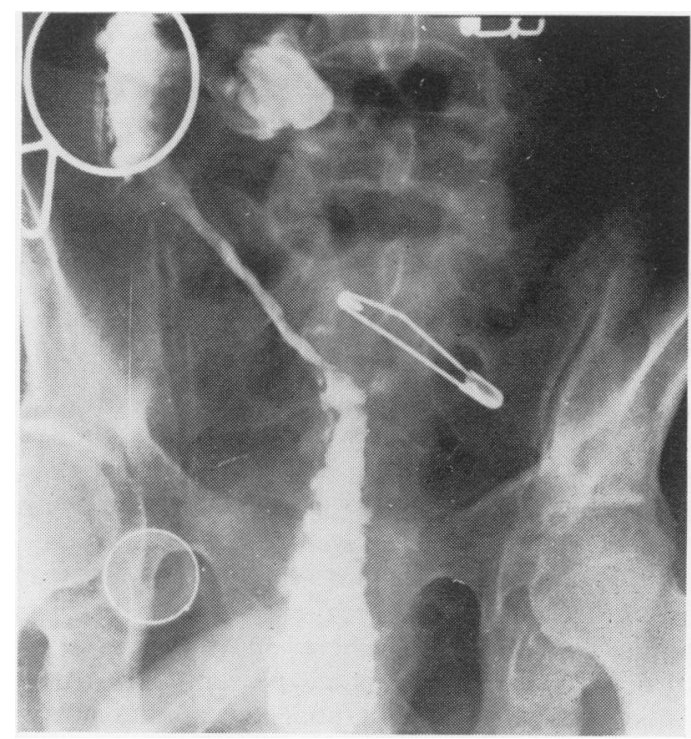

Fig. 2 Barium enema one month after subtotal colectomy in case 1 showing the rectum to be a fibrotic tube with ulceration. The fistulous tract on the left of the photograph resolved but the rectum remained unchanged on subsequent examinations. recovery. The pus from the abscess cavity became less in quantity and the drain was ultimately removed. The rectal stump had discharged large quantities of pus after operation and was irrigated throughout his stay in hospital with a solution of kanamycin. Six weeks after the operation he no longer had any discharge although sigmoidoscopic examination of the rectal stump revealed a tube of granulation tissue with no evidence of any residual normal mucosa. Biopsy of the rectal stump at this stage showed granulation tissue only. There was no evidence of any active amoebiasis. Barium enema was performed (Fig. 2) and this revealed a distorted tube of granulation tissue with several collar-stud abscesses. It was surprising how little disability the patient had from his grossly abnormal rectal remnant.

The patient was discharged from hospital and was fitted with an ileostomy bag on 28 October 1968. He was subsequently seen in the Outpatient Department one year after his discharge from hospital and was perfectly fit and well. He had no problem from the rectal stump and he was coping perfectly satisfactorily with the ileostomy. The fistulae on the abdominal wall had all closed.

\section{Comment}

There are several points of importance in this case. (1) Despite the florid amoebic colitis, examination of the stools and rectal slough failed to demonstrate any active amoebae. (2) While it is possible that early anti-amoebic therapy at the time of admission may have averted the necessity of surgery, the finding of an impending perforation at the rectosigmoid junction as well as several other patches of necrosis of the rectum and sigmoid colon makes this unlikely. Colectomy would appear to have been the only procedure that could have saved this man's life. (3) Despite anti-amoebic therapy, the ascending colon and terminal ileum had already sloughed and this required removal with a secondary procedure. Examination of the pathological specimen suggested that recovery would have been impossible had the colon remained in situ. Microscopically there was hardly any evidence of viable mucosal tissue and gangrenous patches extended through to the serosa in several areas. (4) Marked involvement of the terminal ileum was present, a finding which is not uncommon in the more severe cases at necropsy. (5) The drainage of a liver abscess six weeks after treatment with a full course of emetine hydrochloride and chloroquine diphosphate revealed the presence of active amoebic organisms. A further course of emetine was ineffective but there was a dramatic response to metronidazole. (6) The fibrosis and contracture of the rectal stump, still present many months after colectomy, suggested that complete regeneration of the colonic mucosa would have been virtually impossible. It is likely that the 
patient would have been left with a severe postamoebic colitis.

\section{CASE 2}

A coloured female aged 23 years had diarrhoea (six stools per day) with blood and mucus for three weeks before admission. For the last eight days she had also had a stabbing pain in the right upper quadrant with bouts of nausea and vomiting. She had lost a great deal of weight. She was seven months pregnant. On examination she appeared ill. Her temperature was $100^{\circ} \mathrm{F}$ but she was not dehydrated or jaundiced. The pulse rate was 100 per minute and there was a systolic murmur at the cardiac apex. The blood pressure was $110 / 60$. The chest and central nervous system was normal. Palpation of the abdomen revealed a uterus that was 30 weeks in size and the foetus was lying as a breech. The liver was not enlarged but there was a tender, well defined mass in the right upper quadrant just lateral to the fundus of the uterus. The urine contained a trace of protein and trace of bilirubin, the haemoglobin was $12 \mathrm{~g} \%$, the white cell count $20,500 \mathrm{~mm}^{3}$, and the ESR $80 \mathrm{~mm} /$ hour. Sigmoidoscopically the rectal mucosa was inflamed and much blood and mucus were seen. A small ulcer was visualized and a swab revealed red blood cells, pus cells, and dead vegetative amoebae. In view of the pregnancy, emetine was not given but the patient received $800 \mathrm{mg}$ metronidazole three times daily, rolitetracycline (Reverin), $275 \mathrm{mg}$ intravenously, and chloroquine diphosphate, $500 \mathrm{mg}$ twice daily.

The patient appeared to be responding to treatment and the temperature gradually subsided. The pulse rate subsided to 80 per minute, but 48 hours after admission, she suddenly developed severe generalized abdominal pain. Examination at that stage revealed guarding and rigidity of the whole abdomen and the mass was no longer palpable.

Within six hours of the acute episode, laparotomy was performed. Exposure was obtained by a right subcostal incision (in view of the large uterus) and this revealed a large, free perforation in the ascending colon just below the hepatic flexure. The hole had been plugged by large sheets of necrotic tissue and there was soiling of the peritoneal cavity. The regional colon was oedematous and particularly friable around the perforation. The perforated bowel was exteriorized by bringing it out through the wound and retained in position with a colostomy rod. Inspection of the lumen of the exteriorized bowel revealed large necrotic ulcers with normal intervening mucosa. Biopsies were taken. Several drains were inserted into the peritoneal cavity. Histology showed necrotic tissue and exudate with an extreme degree of autolysis, corresponding to that usually associated with amoebic colitis. While this appearance was suggestive of amoe- biasis, it was not complete confirmation in the absence of amoebae which had not been demon-?

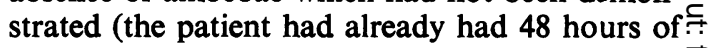
anti-amoebic therapy). Another specimen of $\overrightarrow{\vec{c}}$ : bowel showed non-specific chronic inflammatory $\frac{5}{0}$ infiltrate. A small vessel in the submucosa con- $\frac{C}{\sigma}$ tained a recent thrombus.

The patient made a satisfactory postoperative $\frac{0}{\bar{D}}$ recovery and emetine, 1 grain daily, and chloro- $\frac{\varrho}{\otimes}$ quine, $300 \mathrm{mg}$ twice daily, were given intra- $\omega$ muscularly. As soon as the ileus had subsided, $\overrightarrow{0}$ metronidazole, $800 \mathrm{mg}$ three times daily, was commenced orally. Thirty-six hours after the $\vec{\sigma}$ operation the patient delivered spontaneously of $\stackrel{\varrho}{\varrho}$ a 30-week breech baby which subsequently died $\subsetneq$ from pulmonary complications. During the $\vec{\square}$ recovery phase from the first operation, sigmoidoscopy through the exteriorized colon was $\overrightarrow{0}$ performed and regeneration of the ulcers was $\nexists$ visualized.

The patient maintained a steady postoperative recovery. The stools were particularly loose and $Z$ control of the colostomy was difficult, but at no stage was there any digestion of the skin. She $\frac{\Phi}{3}$ was ultimately discharged from hospital in a good $\underset{\varnothing}{\varnothing}$ condition and was re-admitted six weeks later $\vec{\bullet}$ when the exteriorized colon was resected and an end-to-end anastomosis was performed. It was. interesting to note that the mucosa had regenerated completely but where there had been particularly large ulcers, there was loss of mucosal folds.

\section{Comment}

Extension of a suspected colonic perforation occurred while the patient was receiving anti-? amoebic therapy and appeared to be showing a gratifying clinical response. Exteriorization of the perforated area of the colon was undertaken and this was followed by resection of the exteriorized segment and end-to-end anastomosis.

\section{Discussion}

Although amoebic colitis is a mild disease in $\stackrel{N}{N}$ most patients, and readily cured by anti-amoebic measures, it occasionally presents as a severe or even fulminating illness. Mortality figures are $\stackrel{\varrho}{\Phi}$ not available for acute amoebic colitis, but $\cong$ Wilmot (1950) estimates it at about $3 \%$. When the frequency of amoebiasis in South Africa and elsewhere in the world is considered, this $\underset{D}{\mathbb{D}}$ estimated mortality for amoebic colitis is quite $\frac{\rho}{\mathbb{D}}$ substantial and second probably only to deaths $\cong$ due to amoebic liver abscess. Furthermore, most deaths occur in the severe or fulminating variety 8 of the disease so that this extreme form probably accounts for nearly all of the $3 \%$ of deaths. The incidence of fulminating colitis in all patients with 
amoebic colitis is also not known. It can be assumed that the frequency is low, as many patients with mild colitis are treated on an outpatient basis, and a further group probably responds fairly adequately to simple antibiotics without diagnostic proof of the amoebic aetiology of the bowel upset.

A review of the causes of death in these severe cases is fundamental to our suggested policy of treatment. In a necropsy series reported by Kean, Gilmore, and van Stone (1956) the most common finding was peritonitis and there were $30.4 \%$ perforations in 148 fatal cases. This must indicate that the patients either entered the hospital in a moribund condition with free or impending perforation or that medical treatment was ineffective in controlling the disease before death. Despite this finding the authors fail to mention colectomy, or indeed, abdominal surgery in their section on treatment.

This fulminating form of the disease is usually characterized by severe bloody diarrhoea, often with mucosal sloughs, extreme toxaemia with high pyrexia, tachycardia, leucocytosis and sedimentation rate, or alternatively with a lack of homeostatic mechanisms with toxaemia, only a slightly elevated temperature, leucocyte count, and sedimentation rate. In either case, the most important diagnostic and prognostic signs are found on abdominal examination. Abdominal distension and/or tenderness should alert one to the potential severity and possibly fatality of the outcome. These signs may be present on admission or develop during the course of medical treatment.

Abdominal distension may be due to (1) paralytic ileus caused either by toxaemia, peritonitis, or electrolyte imbalance, or (2) toxic megacolon as found in ulcerative colitis. Localized or generalized abdominal tenderness suggests that the inflammatory process has traversed the muscular layer and provides a pointer to subserosal extension and potential or actual perforation of the bowel. An abdominal radiograph may reveal either subdiaphragmatic air (which is rare), small and large bowel distension with or without airfluid levels, or dilatation of the large bowel alone, the latter being characteristic of toxic megacolon.

It is our contention that surgical intervention may become necessary at any stage in this form of the disease, and the results of the two patients reported above suggest that this is a feasible proposition with modern medical care and the wide variety of anti-amoebic measures now available. Our policy at present is to institute intensive medical measures consisting of nasogastric suction, intravenous therapy, correction of acidosis, hypokalaemia, and other electrolyte imbalance and, in addition, the administration of anti-amoebic treatment and antibiotics. Frequent re-assessment of the patient's clinical status is mandatory with special attention to the abdominal signs. Although each individual case should be judged on its merits, increasing distension, and/or tenderness despite electrolyte correc.ion and anti-amoebic treatment, warrants the serious consideration of surgical intervention. A workable approach might well be that (1) medical measures should be continued if the patient's general and abdominal signs improve progressively or do not deteriorate. (2) Emergency surgery should be seriously considered if the general and local or radiological signs get worse after 24 or 48 hours despite electrolyte correction and anti-amoebic measures, or if acute episodes occur, ie, sudden, increasing distension or acute abdominal pain with increasing tenderness. Continued 'watching' of the patient under these circumstances may result in a situation where the patient is too ill for surgery to be undertaken. (3) Where the physical signs remain static and profuse diarrhoea continues, with difficulty in maintaining electrolyte balance despite intensive therapy after five or more days, and no other cause for diarrhoea can be found, surgery should be contemplated. Amoebae can often no longer be found in the stools but mucosal sloughing continues.

\section{SURGERY IN ABDOMINAL AMOEBIASIS}

While there are few indications for surgery in amoebic colitis, the following conditions may be advanced for consideration.

(1) A liver abscess which fails to respond after repeated needle aspiration and a combination of emetine, chloroquine, and metronidazole. This may require formal laparotomy and drainage (Rivers, Heibner, and Powell, 1955).

(2) Perforation of the bowel with localized abscess formation not responding to medical measures will require open drainage, and

(3) free perforation of the colon with intraabdominal soiling. There would appear to be no place for conservatism under these circumstances (Wilmot, 1962). Emergency laparotomy should be undertaken and if the disease appears to be localized to one segment of the bowel, then the perforated segment should be exteriorized or excised and a proximal colostomy performed (Theron, 1947). After recovery, re-anastomosis should be carried out.

(4) Fulminating amoebic colitis (as described) failing to respond or becoming worse on intensive medical therapy. If laparotomy shows multiple areas of sloughing, gangrene, or impending perforation, subtotal colectomy and colostomy with retention of the rectum should be carried out. Although Palaez et al (1966) mentioned this form of treatment, they state that the patient is usually too ill for surgery. Clearly one should operate before the patient's condition makes surgery a hopeless proposition. If after treatment, the rectum returns to normal, an ileorectal anastomosis may be carried out.

(5) In cases of severe postamoebic colitis, that is, those patients in whom medical measures 
have succeeded in eradicating the amoebae but the patient is left with an 'ulcerative colitis-like' state with anaemia, hypoalbuminaemia, and a raised sedimentation rate. If the clinical course is progressively downhill, the same surgical indications as for severe ulcerative colitis should apply (Powell and Wilmot, 1966).

We should like to thank DrI. N. Marks for helpful criticism and advice and Professor J. H. Louw for permission to publish.

This study was supported in part by the Council for Scientific and Industrial Research and the Medical Research Council of South Africa.
References

Barker, E. M. (1958). Colonic perforation in amoebiasis $S$. Afr. med. J., 32, 634-638.

Cope, Z. (1920). Surgical aspects of dysentery. Lancet, 1, 579-585.

Evans, T. C. (1925). The surgical aspects of amoebiasis. Trans. roy. Soc. trop. Med. Hyg., 19, 282-311.

Kean, B. H., Gilmore, H. R., Jr., and van Stone, W. W. (1956). Fatal amoebiasis: Report of 148 fatal cases from Armed Forces. Institute of Pathology. Ann. intern. Med., 44, 831-843.

Ochsner, A., and Debakey, M. (1942). Surgical amoebiasis. Int. Clin., 1, 68-99.

Peláez, M., Villazón, A., and Zaraboso, R. S. (1966). Amoebic perforation of the colon. Dis. Colon. Rect., 9, 356-362.

Powell, S. J., and Wilmot, A.J. (1966). Ulcerative post-dysenteric $\vec{O}$ colitis. Gut, 7, 438-443.

Rives, J. D., Heibner, W. C., and Powell, J. L. (1955). The surgical complications of amebiasis of the colon (exclusive of liver abscess). Surg. Clin. N. Amer., 35, 1421-1426.

Theron, P. (1947). Surgical aspects of amoebiasis. Brit. med. J., 2, 123-126.

Wilmot, A. J. (1950). Clinical manifestations of amoebiasis in the Bantu. D.M. Thesis, University of Oxford.

Wilmot, A. J. (1962). Clinical Amoebiasis, p. 39. Blackwell, London. 